\title{
La communication dans le travail
}

Intervention faite dans le cadre d'une formation de l'AFCI (Association française de communication interne), le 18 mars 2010

\section{Philippe Zarifian}

\section{OpenEdition}

\section{Journals}

Édition électronique

URL : http://journals.openedition.org/communicationorganisation/1462

DOI : $10.4000 /$ communicationorganisation. 1462

ISSN : $1775-3546$

Éditeur

Presses universitaires de Bordeaux

\section{Édition imprimée}

Date de publication : 1 décembre 2010

Pagination : 135-146

ISBN : 978-2-86781-743-4

ISSN : 1168-5549

Référence électronique

Philippe Zarifian, « La communication dans le travail », Communication et organisation [En ligne], 38 |

2010, mis en ligne le 01 décembre 2013, consulté le 10 décembre 2020. URL : http://

journals.openedition.org/communicationorganisation/1462 ; DOI : https://doi.org/10.4000/ communicationorganisation. 1462 


\title{
La communication dans le travail01
}

\author{
Philippe ZARIFIAN ${ }^{02}$
}

\section{Introduction}

Je voudrais en introduction, avant d'aborder ces deux thèmes, vous proposer une première définition, très basique, très élémentaire, de ce que l'on peut entendre par « communiquer».

Communiquer, c'est se parler à propos de quelque chose. On pourrait ajouter: c'est s'écrire, bien que les personnes s'écrivent de moins en moins. Cependant on peut considérer que les messages par mail, dès qu'ils entrent dans un échange, une discussion, sont une manière de s'écrire. Même chose pour la participation à un Forum ou un réseau social. Néanmoins, la matrice reste bel et bien «se parler» (et non pas «parler»).

On peut se parler selon différents modes:

- l'usage de la parole, la formulation d'énoncés, empruntant aux ressources d'une langue, comprise par ceux qui communiquent.

- l'usage de gestes: dans le travail, ces gestes apparaissent nécessaires lorsque le niveau de bruit dans un atelier ou l'éloignement sont trop importants pour s'entendre. Il est structurellement important dans le modèle du métier, lorsque l'apprentissage des bons gestes apparaît central: non seule-

01. Intervention faite dans le cadre d'une formation de l'AFCI (Association française de communication interne), le 18 mars 2010.

02. Philippe Zarifian est diplômé de Sciences Po Paris, docteur en économie et habilité à diriger des recherches en sociologie. II occupe actuellement la fonction de professeur des universités en sociologie à l'Université Paris-Est Marne-la-Vallée. Après avoir accompli la mission de créer une filière entière d'enseignement de la sociologie dans cette université, il assure actuellement la codirection du master 2: Compétences et Organisations. Ses recherches portent sur les questions de mutation du travail et des métiers, de transformation des organisations (de l'organisation du travail à l'organisation globale d'une entreprise), de pilotage et d'accompagnement dans l'introduction d'une "logique compétence " en entreprises et administrations. II a publiē de nombreux ouvrages, dont le plus récent: Philippe Zarifian, Le travail et la compétence: entre puissance et contrôle, éditions PUF, mai 2009

Site personnel: http://philippe.zarifian.pagesperso-orange.fr/ 
ment on enseigne les gestes de métier à l'apprenti, mais les gens du métier s'accordent entre eux sur les bons gestes en les faisant.

- L'usage, tout à fait important, des expressions du visage. Il se trouve que les êtres humains disposent de muscles du visage, qui autorisent une grande diversité d'expressions. C'est un mode essentiel pour le partage social des émotions, leur communication.

Ce «se parler» peut se réaliser en coprésence physique des interlocuteurs, mais aussi à distance. Incontestablement, dans la vie sociale d'aujourd'hui les communications à distance ont pris une grande place. Mais on voit aussitôt que les deux modes: gestes et expressions du visage, soit disparaissent, soit leur transmission (par vidéo par exemple) est de médiocre qualité. Une partie des émotions peut passer, mais uniquement par le timbre de la voix. La coprésence physique est clairement supérieure pour une communication de qualité et réussie. Elle peut bien entendu alterner avec des communications à distance.

Cette proposition de définition, bien que très basique et intuitive, marque une nette différence entre "communiquer » et « informer». Dans la communication, dans le fait de se parler, sont engagées deux caractéristiques majeures:

- la réciprocité: se parler, et avec elle la relance de l'échange langagier, le dialogue au sens rigoureux du terme;

- la compréhension d'autrui. D'abord bien entendu la compréhension minimum de la signification de ce qui est dit (parler à peu près la même langue et mettre les mêmes réalités derrière les mots), mais aussi un minimum de compréhension de l'autre: sa culture de référence, sa manière de réagir dans une discussion, son adhésion à ce qui est dit ou son rejet, etc. Tous ces aspects sont, de fait, présents dans cette simple action qu'est "se parler».

Je montrerai que la compréhension réciproque, l'intercompréhension, représente une dimension décisive de la communication, mais cela me demandera d'entrer bien davantage dans le contenu de ce que l'on peut entendre par « se comprendre».

\section{L'importance prise par la communication dans le travail de nos jours.}

Elle est largement inscrite dans deux mouvements:

- Un mouvement interne à l'organisation: la nécessité de coopérer et/ou de développer le travail collaboratif. Coopération en équipe, en ligne, en projet, en processus, en réseau... Il existe aujourd'hui une diversité de formes organisationnelles portant la coopération dans le travail, mais la tendance principale est de développer des coopérations inter-métiers et inter-fonctions.

- Un mouvement que l'on peut qualifier de sociétal: un fort besoin de créer ou de recréer de la société, de « faire société » comme le disait le sociologue Georg Simmel, en réaction au constat de désintégration des liens sociaux 
et d'une perte de solidarité. Avec le constat d'un isolement croissant des personnes, malgré et dans la densité des contacts possibles. Dans les entreprises, cela s'exprime par une demande sourde de briser l'isolement, de trouver de la chaleur humaine, d'avoir des visées et perspectives communes, de cultiver l'amitié contre la mise en concurrence.

Je voudrais apporter des précisions sur chacun de ces deux aspects.

\section{Qu'est-ce que coopérer?}

Coopérer, c'est opérer ensemble, agir ensemble, travailler conjointement, et cet agir ensemble ne peut se consolider, en entreprise, que si l'on partage des enjeux communs. Des enjeux, plus que des objectifs. Les enjeux sont à plus longue portée que des objectifs précis à un moment donné. Et pour affronter les enjeux, il faut les connaître, en développer l'intelligence. Un enjeu ne peut pas être prescrit. Il doit être compris et admis. Qu'est-ce qui fait enjeu pour le devenir de l'entreprise? Et pour une activité professionnelle commune?

C'est pourquoi j'ajoute: agir ensemble en connaissance de cause:

- soit en partageant directement une action et sa signification,

- soit en s'organisant ensemble au préalable pour faire converger les actions vers un même effet, quitte à opérer conjointement des réajustements pour vérifier cette convergence et garder la pertinence des actions par rapport aux enjeux.

Ce qui marque le plus la période actuelle et qui est souvent négligé par le management, c'est que le vecteur principal de la coopération est précisément la communication directe dans le travail (et pas simplement le cadre organisationnel ou l'action managériale).

Pourquoi?

À cause de la complexification, de l'intellectualisation, de l'évolutivité rapide du travail à réaliser en commun. Il faut partager la compréhension des problèmes, faire se confronter leur analyse, se projeter ensemble dans l'avenir et anticiper les actions à mener, voire: il faut co-élaborer, coécrire en quelque sorte la conception de que l'on doit entreprendre ensemble. Du même coup, on vérifie empiriquement que toute coopération suppose des temps d'échange, de discussion, de réflexion collective, pour se mettre d'accord sur la compréhension des problèmes à résoudre, pour se réajuster, pour rectifier, pour mettre en commun l'expérience acquise, pour explorer de nouvelles solutions, etc. C'est cela le travail d'aujourd'hui et plus encore: de demain. Mais ces temps manquent aujourd'hui cruellement dans les entreprises. On n'a pas encore pris conscience de leur importance. Le taylorisme continue à marquer les esprits.

Je résumerai les choses en disant que travailler devient: prendre des initiatives ensemble ou convergentes, les plus pertinentes possibles au regard des enjeux. Donc, du point de vue de l'organisation du travail, c'est assembler et composer ensemble des compétences (sachant que pour moi le cœur de la compétence réside dans la prise d'initiative et de responsabilité face 
à des situations productives évolutives et de plus en plus marquées par des événements imprévus)03. Ce mode de coopération et donc de communication est très frappant dans les organisations par projet. Mais, si on veut éviter une montée inconsidérée du nombre des projets, qui risque de fragmenter le corps social et de complexifier l'organisation, il serait bon que cela pénètre l'organisation ordinaire du travail.

\section{Qu'est-ce que faire société? Et en quoi la communication y contribue?}

C'est une question plus difficile que la précédente. On bénéficie en sociologie de deux traditions théoriques différentes pour aborder cette question. La première vient de Durkheim = la société est avant tout une question d'ordre, de cohésion, de solidarité. Le point essentiel de l'intégration sociale est représenté par les règles sociales (sociétales) de comportement moral auxquelles chacun doit accepter de se plier et d'intérioriser. Faire société, c'est alors trouver ou retrouver les règles et valeurs morales (qui relèvent de ce qui est bien ou mal de faire) qui permettent d'unifier les individus concernés. Une règle par définition est une contrainte, mais bien entendu c'est une contrainte, un impératif d'autant plus fort que les individus l'intériorisent dans leur psychisme. Ces règles et valeurs sociales transcendent, dépassent les motifs et intérêts individuels.

La seconde tradition vient de Max Weber. Il insiste sur la question du sens de l'activité sociale dans laquelle chacun est engagé. Le sens, pour Weber, ce sont les motifs et les raisons que chacun a d'agir, dans une activité sociale, donc en interaction avec autrui. Motifs: les finalités qu'on s'assigne et qu'on poursuit. Raisons: la manière dont on raisonne, de façon rationnelle, son engagement dans l'activité. Les "raisons de » se comporter de telle ou telle manière. J'ajoute, à titre personnel, que le sens, c'est aussi et surtout l'orientation que l'on donne à son action, la perspective quant au devenir. Un devenir tendu entre la mémoire du passé et l'anticipation du futur, au présent des actions engagées. Des devenirs: à tout moment, s'offre une palette de devenirs possibles. Chaque action est un choix en faveur de tel ou tel devenir, que l'on en ait conscience ou non.

Dans ce cas, faire société, ce n'est pas principalement respecter des règles communes, mais c'est s'accorder ensemble sur le sens accordé aux actions et aux comportements et ceci à partir d'une compréhension réciproque du sens que chacun $y$ place. Lindividu, dans son épaisseur personnelle, n’est ni nié, ni dépassé, ni transcendé. Chaque individu engage, tout ou partie de soi, en s'accordant et en s'engageant autour d'un sens commun. J'insiste sur le mot « commun » et non " général ». Le commun émerge du singulier, alors que le général est supposé encadrer et absorber le particulier. Et pour être véritablement commun, il faut en parler, en discuter, échanger à son propos. Bref: il faut communiquer. On retrouve le «se parler » que j’ai mentionné au début. Sans nier la nécessité de

03. Voir Philippe Zarifian, Le modèle de la compétence, éditions Liaisons, septembre 2004. 
la tradition ouverte par Durkheim, $j$ 'adhère, en tant que sociologue, beaucoup plus à la tradition issue de Max Weber, qualifiée de « sociologie compréhensive » et je pense qu'elle est plus adéquate pour penser la société actuelle. En entreprises, je pense personnellement que la vision de type durkheimienne est beaucoup trop présente, avec toujours un risque de confusion entre des règles vraiment sociales et des prescriptions managériales. L'ordre, certes, mais quel ordre? Par contre la construction d'un sens commun fait souvent défaut. C'est un sérieux problème.

Compte tenu de ces deux traditions d'analyse, la question du «faire société » est devenue particulièrement importante aujourd'hui à cause des effets de dislocation sociale de la dernière période. Par exemple:

- La crise du taylorisme a eu un effet qu'on ne souligne pas assez: elle a révélé, « à nu », une absence de sens donné au travail. L'absence de sens n'est pas nouvelle: elle était inscrite au cœur du taylorisme. Mais ce qui est nouveau, c'est qu'elle n'est plus masquée par le travail à accomplir sous prescription. Dès lors que, soit les individus se trouvent exclus, en situation de chômage, soit la prescription recule, la question du sens donné à son travail éclate au grand jour! Le vide apparaît.

- La crise de la fermeture des métiers sur eux-mêmes. Pour des multiples raisons, les métiers ne peuvent pas perdurer dans et par leur fermeture, qui, quand elle s'exacerbe, apparaît, à juste titre, pour du corporatisme. Leur ouverture oblige à dépasser le sens purement interne qui existe dans une communauté de métier, à se risquer dans une coopération inter-métiers, à se replacer dans les enjeux et l'organisation globale de l'entreprise.

- La crise des découpages fonctionnels qui oblige, par exemple, le vendeur à tenir compte ce que la production peut offrir et inversement. Cette crise, trop peu mentionnée à mon avis, interrogent les finalités et l'orientation du travail. Il faut penser "global " et non plus uniquement de façon sectorielle. Qualité globale, délai global, service global offert et rendu aux clients, etc. Il ne faut pas prendre ces termes pour des effets de mode venu du Japon. Ils désignent une nécessité profonde.

- Enfin, en arrière-plan, il existe tous les effets de la dislocation et multiplication des statuts d'emploi, la forte montée de la sous-traitance, tous phénomènes qui divisent les salariés, engendrent des pertes de solidarité.

\section{Reconstruire du sens commun devient prioritaire.}

\section{Problèmes nouveaux}

À période nouvelle, problèmes nouveaux. Ces dernières années, deux problèmes nouveaux ont pris de l'importance, qui sont venu à la fois perturber et enrichir la question de la communication. 


\section{Les emplois de relation clients ou publics}

Le premier est l'importance prise, y compris au plan quantitatif, par les emplois de la relation directe avec les clients, soit en face à face, soit par téléphone. Clients, publics ou usagers. On pourrait y ajouter en effet des emplois nombreux qui existent dans les administrations, comme ceux que l'on trouve dans les services de la sécurité sociale ou tout simplement ceux de l'éducation nationale. Ces derniers emplois ne sont pas nouveaux. C'est plutôt leurs conditions d'exercice qui le sont. Dans ces emplois, on peut affirmer: travailler, c'est très principalement communiquer, et donc, si possible, savoir le faire, de manière professionnelle. La communication et sa réussite sont au cœur du travail, bien qu'elles ne soient pas toujours au cœur de l'évaluation (pensons aux centres d'appel téléphoniques lorsqu'ils restent principalement évalués sur des débits d'appels!).

Cela soulève une série de questions.

D'abord on constate que ce sont des activités professionnelles qui s'exercent dans une relative solitude. Il peut certes y avoir des entraides, des lignes dites de « soutien » sur les produits ou procédures, l'appui d'un collectif, mais il faut constater, sur la durée, que ces collectifs ont plutôt eu tendance à s'affaiblir et que, pour l'essentiel, le professionnel est seul face au client ou au public. Le guichetier, seule face au client qui sort de la file d'attente, l'opératrice, seule au téléphone (avec, par exemple, un client agressif), l'enseignant seul face à une classe. Cela induit un effet qu'il ne faut pas négliger: ce sera à lui de répondre entièrement de la situation de communication, de sa réussite ou de son échec. En dernière analyse, qu'elle que soit l'aide qu'il reçoit, il est seul responsable, côté entreprise ou administration. Il doit assumer l'entièreté de la situation. Selon mes observations, peu d'encadrant en tiennent compte. Mais dire que ces salariés sont seuls est bien entendu un paradoxe. Ils ne sont pas seuls puisqu'il y a au moins deux personnes en face à face, voire nettement plus. Mais entre eux et les clients ou publics, les rôles ne sont pas symétriques et la communication qu'ils nouent n'est pas du même type que celle interne à l'entreprise. Elle ne relève principalement, ni de la coopération, ni du " faire société ", bien que ces deux dimensions ne soient pas totalement absente. Pour l'essentiel, cette communication professionnelle relève de la prestation de service. Cette communication, d'après mes analyses, oscille entre deux attitudes possibles, deux pôles:

- Le pôle de la communication instrumentale, étroitement finalisée : il s'agit de placer un produit, voire, parfois, de « placer des connaissances ou des renseignements " sans se préoccuper des attentes et compréhensions réelles des interlocuteurs. Dans l'univers marchand, il faut placer un produit ou un service et provoquer une dépense de la part du client. C'est ce que les vendeurs eux-mêmes appellent de la "vente forcée ». Mais de quoi parle-t-on? S'agitil vraiment de communiquer, au sens où j'en ai parlé jusqu'à maintenant? 
Non. Dans l'usage d'un langage instrumental, une impasse est réalisée par rapport au fait de «se parler». On ne cherche pas à comprendre autrui. On exerce sur lui un pouvoir, l'espace de la communication n'étant pas autre chose que l'espace d'exercice de ce pouvoir avec l'usage de techniques connues (les techniques de vente par exemple). Je n'ai pas à juger de cette pratique. Il faut simplement dire que l'usage du terme " communication » serait ici abusif.

- Le second pôle privilégie la compréhension de ce quattend un client, voire des besoins qui se sont fait jour dans son mode de vie, et qui l'ont conduit à contacter l'entreprise. Attitude compréhensive, qui, vous le voyez, renvoie à ce que j'ai déjà dit sur l'intercompréhension, comme élément central de la communication, à cette différence près que la compréhension est surtout du salarié vers le client et moins l'inverse. On retrouve les vertus de l'écoute, du respect réciproque, l'importance de laisser le temps de s'exprimer complètement, voire d'encourager et aider cette expression par des petites questions appropriés. Il s'agit alors moins d'une technique que d'un art (et on parle bel et bien de l'art de la discussion, l'art de la conversation). Néanmoins, même ainsi considérée, cette communication compréhensive ne se confond pas avec la communication interne à l'entreprise. Parce qu'entre les interlocuteurs, l'univers de référence n'est pas identique. Du côté du client ou de l'usager, on trouve tout son univers de vie sociale et personnelle, qui se condense à un moment donné dans la demande de tel service. Du côté du salarié, on trouve l'univers de l'entreprise ou de l'administration qui peut offrir tel ou tel service, tout le back-office, et, bien entendu, les conditions d'exercice de son travail en tant que salarié.

Je propose alors de dire que la communication, dans la prestation de services, réside dans la rencontre entre deux univers hétérogènes et la communication réussit lorsque le point de rencontre pertinent est cerné et lorsque le service (ou le potentiel de service) qui va entrer dans l'univers du client s'avérera utile, « donnant satisfaction ", selon la formule consacrée. Il est, bien entendu, passionnant pour un chercheur de voir comment les deux types de communication (communication interne à l'entreprise et communication en direction de personnes externes) se combinent ou non! Mais vous en parler m'entraînerait trop loin! Donc, au total, deux attitudes différentes, deux sortes d'agir (instrumental et compréhensif), avec parfois un mélange des deux ou une hésitation entre les deux.

\section{La généralisation du contrôle par objectifs/résultats}

C'est le second phénomène marquant de la période actuelle. Cela renvoie au mode de contrôle du travailleur et à son évaluation (évaluation de sa performance, davantage qu'évaluation de sa compétence). Je serai très bref sur ce sujet car il demanderait de nombreux développements. Je ne peux que renvoyer à mon dernier livre (Le travail et la compétence: entre puissance et contrôle, éditions PUF, mai 2009). Disons, pour aller à l'essentiel, que le 
contrôle instauré par fixation d'objectifs et évaluation de résultats a tendance à tuer toute communication authentique. Le travail, ses difficultés, son intérêt, ses potentialités, son sens, disparaissent, deviennent invisibles. N'apparaissent que les objectifs et résultats, souvent réduits à des chiffres. Il n'existe pas de communication. On ne se parle pas. L'échange de paroles, au moment de l'évaluation des résultats et/ou des performances, revient à l'exercice d'un pouvoir dissymétrique, ce qui ne laisse que peu de place à la compréhension réciproque. En parallèle, je l'ai dit, on assiste à une mise en disparition du travail et de sa qualité, donc de ce qu'il y a de plus important pour le salarié. Dans un cas-limite, si on veut pratiquer l'humour noir, on devrait pouvoir automatiser ces rencontres et entretiens. On les remplacerait par un dispositif, ayant en input les fameux objectifs (si possible personnalisés) et en ouput une évaluation chiffrée du résultat et l'appréciation, édités automatiquement...! Il serait pourtant combien plus facile et enrichissant de profiter de ces entretiens pour s'entretenir... du travail réel, des situations rencontrées et des compétences que ce travail requiert pour être de qualité (avec confrontation entre vision de l'entreprise et vision du salarié)! Quand on ne discute plus du travail réel et de ses conditions de réussite, du sens du travail utile et bien fait, on pratique un grave déni du professionnalisme des salariés.

\section{Qu'est-ce que communiquer?}

M'inspirant du philosophe et sociologue allemand, Jürgen Habermas, connu pour être l'auteur de la théorie de l'agir communicationnel, je dirai que communiquer dans le travail, c'est se mettre d'accord sur quelque chose à faire en commun et agir avec d'autant plus de force que cet accord est profond. Tout se condense alors dans la question: qu'est-ce que se mettre d'accord? D'abord, bien entendu, s'il faut se mettre d'accord, c'est que cet accord n'existe pas ou n'existe plus: soit parce que la situation a changé, ou parce qu'un événement s'est produit, ou qu'une nouveauté est introduite, ou qu'un projet doit être lancé, ou que le « travailler ensemble » englobe désormais des métiers qui ne travaillaient pas ensemble, ou que les attentes du marché se sont modifiées, etc. Bref: les situations «normales » d'aujourd'hui. Puisque l'accord ne préexiste pas, il faut le créer. Et pour se mettre d'accord, j'y reviens, mais à partir d'un autre point de départ: il faut instaurer un véritable dialogue, argumenté, instauré une compréhension réciproque. Habermas distingue trois registres pour instaurer cet accord, registres que je vais reprendre, mais à ma façon, pour les centrer sur la question de la communication dans le travail.

\section{Le registre de la vérité propositionnelle.}

Il s'agit, pour chacun, de prétendre dire le vrai sur un fait ou un événement: sur une panne, sur une réclamation client, sur les conditions de réussite d'un projet, etc. Le vrai n'émerge pas miraculeusement par lui-même. Il faut 
analyser la situation et en discuter, car il est rare que tout le monde ait immédiatement le même point de vue. La communication réussit lorsqu'un accord surgit, après échange de preuves, d'expériences similaires, d'arguments. Bref: après confrontation des intelligences. Bien entendu, dès lors que cet accord suscite une action (pour réparer une panne par exemple), la véritable sanction de la validité de l'accord résidera dans la réussite ou non de l'action. C'est la réalité qui tranche. La machine sera ou non réparée, en visant une réparation durable. Il s'agit ici d'une approche pragmatique, incontournable dans un travail professionnel: on vise en commun le succès pratique.

\section{Le registre des normes morales.}

Qu'est-ce qu'il est bien (et donc mal) de faire? Quel comportement privilégier (entre collègues, vis-à-vis des clients, etc.)? Par exemple, on peut édicter des normes de respect du client, d'honnêteté, d'entraide, etc. On appelle souvent, de manière impropre, ces normes des «valeurs». Non: ce sont des normes et comme toutes normes, elles s'imposent. Mais la communication joue pleinement son rôle lorsqu'il y a débat et choix collectif, raisonné, de telle ou telle norme morale commune. Moins la norme est imposée autoritairement, plus elle aura donné lieu à discussion, plus ensuite elle sera solidement respectée "en connaissance de cause ». On adhère à ce qu'on aura pu discuter voire contester. Ici on vise, non un succès pratique, mais une cohésion sociale, un certain ordre collectif. On voit qu'on rejoint l'approche durkheimienne du « faire société ».

\section{Le registre de l'expressivité personnelle et de l'éthique dans un cadre social.}

Ce troisième enjeu de l'agir communicationnel a été peu développé et, à mon avis, mal analysé par Habermas. Il en a fait le registre de la sincérité ou de l'authenticité. Chacun doit pouvoir exprimer, avec sincérité, ses valeurs et projets de vie bonne, donc son éthique personnelle, de manière à ce que les autres en tiennent compte et que ces éthiques se composent. Cette position de Habermas me semble vraie, mais insuffisante. Je ne pense pas que sincérité et authenticité en soient les caractéristiques principales. Dans ce troisième registre de la communication, il ne s'agit pas de se mettre d'accord. Personne n'a à être d'accord avec l'éthique personnelle de telle ou telle personne. Par contre, il s'agit de l'exercice, difficile mais hautement précieux, consistant à procéder par recoupement, c'est-à-dire à dégager une éthique commune, une même conception de la vie bonne et des vertus qui la portent, sans sacrifier ou dépasser en rien les éthiques personnelles. Et pour cela, c'est moins de sincérité dont on a besoin, mais de liberté et de tolérance. Il s'agit, dans la communication, de comprendre et de tenir compte du sens et des affects que chacun donne à son engagement dans un travail collectif. Mais aussi des difficultés et souffrances personnelles qui font que la vie au travail apparaît douloureuse et affaiblissante et donc nullement «bonne». La définition du 
«bon », dont le modèle paradigmatique reste la santé (qu'est ce qui est bon ou mauvais pour la santé), vient à la fois de ce qui est affirmé en positif et énoncé en négatif. C'est ainsi qu'une éthique commune peut émerger. On est là, on l'aura compris, dans l'approche wébérienne du « faire société ». La condition absolue pour que ce troisième registre puisse s'établir et produire ses effets, c'est que chacun puisse exprimer ses propres valeurs de vie et être écouté. Et réciproquement. Donc être libre de s'exprimer (avec sincérité)... C'est pourquoi je parle à ce propos de liberté et de tolérance, donc de comportements sociaux. C'est le temps de l'expressivité.

Je précise aussitôt, pour éviter toute méprise, que je ne pense en rien aux fameux groupes d'expression. Non seulement l'expression du sens et des affects n'a rien à voir avec ce qui se trouve souvent en jeu dans ces groupes: exprimer une opinion ou une humeur... Mais en plus l'expressivité ici n'est pas tournée vers l'individu qui exprime, mais bel et bien vers le collectif, et plus largement la communauté, pour trouver une éthique commune, une définition commune de la vie bonne (dans le travail). Ce que j'appelle ici «éthique commune » recoupe ce que j'avais appelé "sens commun ", mais elle y ajoute les valeurs de vie. Car c'est bel et bien à propos de l'éthique que la question des valeurs de vie (les seules à être importantes) prend une réelle signification. À valeur, ce à quoi l'on croit et qui suscite notre engagement. À valeur aussi, de manière rationnelle, ce qui engendre une vie bonne, la joie, le plaisir, l'amitié et pourquoi le travail professionnel n'en donnerait-il pas l'occasion? Avoir plaisir à travailler, voilà une proposition simple, élémentaire là encore, mais qu'il faut revaloriser.

\section{Conclusion}

Vous l'aurez vu: je n'ai cessé d'insister sur la compréhension réciproque et sur la production, en connaissance de cause, du sens et des valeurs de vie réellement communes.

Les apports d'une communication réussie sont importants:

- apport central pour la coopération dans le travail et donc pour l'efficience de l'entreprise,

- apport pour le «faire société », pour la cohésion sociale, le dépassement des fractures et désintégrations actuelles, la réunion autour d'enjeux d'entreprise qui soient partagés,

- apport pour produire du sens, individuel et collectif, ce qui impose de pouvoir s'exprimer, être écouté, co-élaborer sens et valeurs de vie communs au travail,

- apport pour l'articulation entre communication interne et communication avec les clients, usagers, publics.

Ce sont des enjeux d'aujourd'hui et plus encore de demain. 
Mais la communication n'est pas aussi spontanée qu'on ne le pense, surtout si l'on tient compte de l'héritage du passé. Elle requiert des conditions matérielles, du temps en particulier. Elle requiert des efforts d'ouverture que chacun doit faire. Elle requiert des organisations qui l'appellent, la nécessitent, en particulier les organisations transverses et par projet. Elle se heurte à des nombreux obstacles. C'est pourquoi je pense personnellement qu'il faut développer, dans les entreprises et administrations, une véritable politique de communication liée au travail, pour en favoriser le développement. Est-ce que la fonction "Communication interne » peut l'autoriser, y inciter, l'impulser? Je ne sais pas. Je vous renvoie la question.

Paris le 21 mars 2010.

\section{BIBLIOGRAPHI}

Zarifian Philippe, Le modèle de la compétence, éditions Liaisons, septembre 2004

Zarifian Philippe, Le travail et la compétence: entre puissance et contrôle, éditions PUF, mai 2009

Adresse mel:philippe.zarifian@orange.fr

Adresse site personnel: http://pagesperso-orange.fr/philippe.zarifian/

Résumé: Communiquer, c'est, dans sa définition la plus simple et la plus essentielle, se parler et essayer de se comprendre. Toute communication implique réciprocité, dialogue. Mais c'est aussi, dans une activité professionnelle, se mettre d'accord sur quelque chose à faire et réussir en commun. Pour cela, il ne suffit pas d'avoir les mêmes objectifs. Il faut partager les mêmes enjeux et donner un sens commun à son engagement dans cette activité. Un sens commun, c'est-à-dire un même motif, une même raison d'agir, et cela ne s'obtient que par une communication intercompréhensive. Dans celle-ci se trouvent engagés, non seulement le souci du collectif, du "faire société », mais aussi une même éthique vis-à-vis de l'action à engager ensemble. Dans les métiers de la relation de service, intervient une autre communication, celle qui se noue avec le client. Elle ne peut être abordée de la même manière, car chaque protagoniste met en avant des préoccupations différentes. L'essentiel alors est que soit créé et organisé un espace particulier, celui dans lequel les deux univers de vie, celui du client et celui de l'agent vont pouvoir de recouper. C'est dans cet espace seulement qu'une intercompréhension pourra être nouée.

Mots clefs: Sens commun, intercompréhension, dialogue, enjeux partagés, accord, relation de service 


\begin{abstract}
Communicate means, in the most simple and essential definition, talking and trying to understand each other. Every kind of communication implies reciprocity, dialogue. But it is also, in an occupation, to come to an agreement and to reach success in common. In order to do that, having the same goals is not enough. It is necessary to share what is at stake, and to give common sense to the activity. A common sense means common motives, common determination, and this can only be achieved thanks to intercomprehension. In this intercomprehension, the concern for the collective, and the feeling of "making society", are not only involved. What is also fundamental is having a common ethics about the action that has to be taken together. In trade service relationships, another form of communication is at stake, that has to do with the customer. It cannot be considered in the same way, since each person puts forward different concerns. The main point then is to be able to create and organize a special area, that allows the the customer and the employee to meet together. It is only in this area that they will be able to meet together, and to build intercomprehension.
\end{abstract}

Key words : Common sense, intercomprehension, dialogue, shared objectives, agreement, trade service relationship 